\title{
Toy Manchester Terrier
}

National Cancer Institute

\section{Source}

National Cancer Institute. Toy Manchester Terrier. NCI Thesaurus. Code C53937.

The Toy Manchester Terrier is a smaller, toy variety of the Standard Manchester Terrier. 